\title{
Band gap reduction in InAsN alloy
}

\author{
Tso-Yu Chu, Hao-Hsiung Lin, and Ding-Kang Shih \\ Room 419, Department of Electrical Engineering and \\ Graduate Institute of Electronic Engineering, \\ National Taiwan University, Taipei, Taiwan, R.O.C. \\ Fax: +886-2-23632442 E-mail: hhlin@cc.ee.ntu.edu.tw
}

\begin{abstract}
We report the absorption and photoluminescence (PL) properties of InAsN alloy grown by gas source molecular beam epitaxy. A calculation based on the band anticrossing model was used to evaluate the Burstein-Moss effect and the band renormalization effect due to the high residual carrier density in the alloy and also the original band gap energy. It can be seen from our calculation that the broad linewidths of the PL spectrums are due to the Burstein-Moss effect, and the high-energy edges of PL spectrum are consistent with the results from absorption measurements. The low-energy edges of PL spectrum are also showed to be close to the calculated original band
\end{abstract} gap energy.

\section{Introduction}

Alloys of low-nitrogen-content group III-V nitride have attracted much attention in the past few years [1-4]. The very large bowing effect on the band gap energy resulting from the large difference in the atomic sizes and electronegativities between $\mathrm{N}$ and other group $\mathrm{V}$ atoms has been demonstrated in several alloys such as InGaAsN[5-6], GaAsN[7-8], GaPN[9], InPN[10], InAsN [11-14], and found lots of important applications on photonic devices. Among the alloys, InAsN is especially promising in the mid-infrared wavelength [14]. In order to know how band gap of $\operatorname{In} A s_{1-x} N_{x}$ changes with the nitrogen composition, $x$, a recently reported model called band anticrossing model was used to analyze the absorption spectrum data of our InAsN samples. This model had been verified on some nitride alloys such as GaAsN[15] and InGaAsN[4,16], but little attention was put on InAsN.

In the analysis based on the anticrossing model, the Burstein-Moss effect [17] and band renormalization effect [18] caused by high residual carrier concentration in our InAsN alloys are also included. The absorption edge of the alloy shows blue shift as the $\mathrm{N}$ composition increases. However, after the consideration of the Burstein-Moss effect and the band renormalization effect, the found original band gap obeys the bowing effect. The PL spectrums of InAsN are also investigated. We show that the broad linewidth of the InAsN alloy is due to the Burstein-Moss effect. Furthermore, the high-energy edge of the spectrum is found to be consistent with the absorption edge, and the low-energy edge of the spectrum is near the original energy band gap from our calculation.

\section{Experiments}

The samples were grown on semi-insulating (100) InP substrates using a VG V-80H GSMBE system. Element
In source and thermally cracked $\mathrm{AsH}_{3}$ and $\mathrm{PH}_{3}$ sources were used for producing molecular beams. The active $\mathrm{N}$ species were generated from an EPI UNI-bulb RF plasma source. For the growth of InAsN, the used RF power was from $200 \mathrm{~W}$ to $480 \mathrm{~W}$ and the range of nitrogen flow rate was from 1.2 to $1.9 \mathrm{sccm}$. Detailed growth conditions are summarized in Table 1 . The oxide of the InP substrates was firstly removed at $500^{\circ} \mathrm{C}$ under $P_{2}$ flux. Then, a $0.3-\mu \mathrm{m}$-thick undoped InP buffer layer was grown at $460^{\circ} \mathrm{C}$ with a growth rate of $1.5 \mu \mathrm{m} / \mathrm{hr}$. A $2-\mu \mathrm{m}$-thick undoped $\operatorname{InAs}(\mathrm{N})$ layer was subsequently overgrown on the buffer layer without a growth interruption and the $\mathrm{AsH}_{3}$ flow rate was fixed at $4 \mathrm{sccm}$. High-brightness mode $\mathrm{N}_{2}$ plasma was ignited for the $\mathrm{N}$-containing growth. The RF power was turned off immediately after finishing the InAsN growth. Besides the InAsN sample, a reference InAs sample was also grown. Their growth conditions were kept the same except the irradiation of the active $\mathrm{N}$ species.

Table 1 Growth conditions and nitrogen composition of InAsN on InP substrate

\begin{tabular}{cccc}
\hline $\begin{array}{c}\text { Sample } \\
\text { No. }\end{array}$ & $\begin{array}{c}\text { RF plasma } \\
\text { power (W) }\end{array}$ & $\begin{array}{c}\text { Flow rate of } \\
\mathrm{N}_{2} \\
\text { (sccm) }\end{array}$ & $\begin{array}{c}\text { Nitrogen } \\
\text { composition (\%) }\end{array}$ \\
\hline C937 & 0 & 0 & 0 \\
C1076 & 300 & 1.2 & 0.5 \\
C1077 & 400 & 1.2 & 1.6 \\
C1117 & 300 & 1.9 & 0.8 \\
C1118 & 300 & 1.5 & 1.2 \\
\hline
\end{tabular}

The structural properties of the samples were investigated using a Bede QCla double crystal X-ray diffractometer (DXRD). The nitrogen composition of the InAsN sample was determined from the DXRD spectra fittings by using a commercial dynamic simulator, 
RADS. Because the film thickness are much larger than the critical layer thickness calculated from the Matthews and Blakeslee's model [19] and the thermal expansion coefficient of InAs is very close to that of InP, the InAsN samples are considered to be fully relaxed in the fitting. The electrical properties of the samples were investigated using Hall effect measurement. In order to determine the absorption coefficient versus photon energy, transmission measurements at near-normal incidence were made using a Bruker IFS 120 HR Fourier transform infrared (FTIR) spectrometer. The measurement was in the range of $1500-11000 \mathrm{~cm}^{-1}$ and with a resolution of $2 \mathrm{~cm}^{-1}$. To calculate the absorption coefficient $\alpha$ from the transmission data we used the procedures described in the literatures [20-21]. In our photoluminescence (PL) measurement system, the 514.5 $\mathrm{nm}$ line of an $\mathrm{Ar}^{+}$laser was used as the excitation source. The samples were placed in a close-cycle helium cryostat for low temperature measurements. The luminescence was detected using a liquid nitrogen cooled Hamamatsu P3357-02 InSb photodiode.

\section{Theories and Calculations}

Band anticrossing model in which localized $\mathrm{N}$ states interact with the conduction band was recently used to explain the band structure properties of the group III-V-N alloys. The interaction between the localized $\mathrm{N}$ state and the conduction band causes the conduction band to split into two sub-bands with dispersion relations given by $[4,15,16]$

$$
E_{ \pm}(k)=\frac{1}{2}\left\{\left[E_{M}(k)+E_{N}\right] \pm \sqrt{\left[E_{M}(k)-E_{N}\right]^{2}+4 V_{N M}^{2}}\right\},
$$

where $E_{M}(k)$ is the conduction band dispersion relation of $N$-free InAs material, $E_{N}$ is the energy of localized $\mathrm{N}$ state, and $\mathrm{V}_{\mathrm{NM}}$ is the coupling element in the following matrix used to derive the dispersion relations of Eq. (1),

$$
\left|\begin{array}{cc}
E-E_{M} & V_{N M} \\
V_{N M} & E-E_{N}
\end{array}\right|=0
$$

All the energies are measured relative to the top of the valence band. We have found that $\mathrm{E}_{\mathrm{N}}$ is about $1.48 \mathrm{eV}$ above the valence band for InAsN. The square of the matrix element is proportional to the nitrogen composition, i.e. $V_{N M}=C_{N M} x^{1 / 2}$, where $C_{N M}$ is a material-dependent constant and $x$ is the nitrogen composition. The localized $\mathrm{N}$ level $\mathrm{E}_{\mathrm{N}}$ was fond to be constant relative to the vacuum level. As can be seen, different nitrogen compositions derive different $E_{+}$and $E$, and $E$. is what we pay attention to. Details of band anticrossing model can be found in other reports [15-16].

If carrier concentration in semiconductors is high, the Fermi level can be above the conduction band bottom by a quantity $E_{F}$. Since the states below $E_{F}$ are already filled, fundamental transitions to states below $\left(E_{8}\right.$ $+E_{F}$ ) are forbidden. The absorption edge should shift to higher energies by $E_{F}$. The quantity of $E_{F}$ can be calculated by solving the following equation,

$$
n=\int f\left(E_{\text {. }}\right) D\left(E_{\text {. }}\right) d E \text {. }
$$

where $n$ is the electron concentration, $f(E-)$ is FermiDirac distribution function and $D(E-)$ is the density of state of InAs(N).

Another effect called band gap renormalization effect also causes band gap to decrease when the carrier concentration increases. This effect is predicted and calculated by the following equation [18]:

$$
\begin{aligned}
\mathrm{E}_{\mathrm{BGR}} & =\left(-\alpha_{\operatorname{InAs}} \mathrm{n}^{1 / 3}\right)\left(\mathrm{m}^{*}{ }_{{ }_{\mathrm{nAA}}} / \mathrm{m}^{*}{ }_{\text {InAsN }}\right) \\
& =\left(-2.671 \times 10^{-8} \mathrm{n}^{1 / 3}\right)\left(\mathrm{m}^{*}{ }_{\text {InAs }} / \mathrm{m}^{*}{ }_{\operatorname{lnAsN}}\right),(\mathrm{eV})
\end{aligned}
$$

where $0_{\ln A s}$ is the renormalization constant of $\operatorname{In} A s$ and the effective mass of InAs and InAsN, $m^{*}{ }_{\text {InAs }}$ and $\mathrm{m}^{*}{ }_{\mathrm{hAsN}}$, are obtained from:

$$
\frac{1}{m^{*}}=\frac{1}{\hbar^{2}} \frac{d^{2} E_{-}}{d k^{2}},
$$

where th is Planck's constant and E- of InAsN is from Eq.(1)

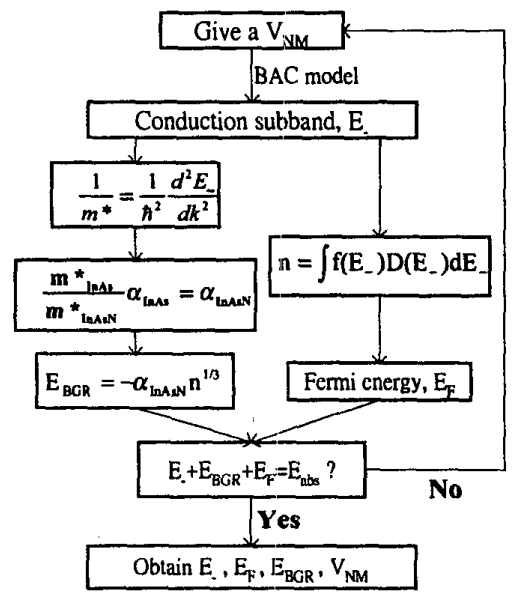

Fig.1 Block diagram of the self-consistent calculation progress.

A block diagram illustrating the overall calculation process is shown in Fig. 1. It is a self-consistent calculation taking band anticrossing model, the Burstein-Moss effect, and the band gap renormalization effect into account. First a test $V_{N M}$ is substituted into $\mathrm{Eg}$. (1), and a conduction subband E- is obtained. Then the E- subband is used to calculate the Burstein-Moss shift (Fermi energy $E_{F}$ ) and the band gap narrowing term, $E_{B G R}$ in two sub-routes. The electron concentration, $n$, is obtained from the Hall effect measurement. If the sum of these three energies, $E-, E_{F}$, and $E_{B G R}$, is not equal to the absorption edge, another $V_{N M}$ will be tested. This loop is repeated until a group of matched energies is obtained. We operated this process on a series of InAsN samples with different nitrogen compositions and got a series of $\mathrm{V}_{N M}$ 's. Finally a linear fitting was done with the plot of $\mathrm{V}_{\mathrm{NM}}$ versus $\mathrm{x}^{1 / 2}$ to get the constant $\mathrm{C}_{\mathrm{NM}}$ of $\operatorname{In} A \mathrm{~N}$ and the result is about 1.68 . 


\section{Results and Discussions}

The nitrogen compositions of our InAsN samples were determined using DXRD measurement and RADS fitting program. Fig.2 shows the square of the absorption coefficient $\alpha^{2}$ versus photon energy plots for these InAs(N) bulk samples. We can note that the energies of the absorption edges of InAsN samples are all higher than that of InAs. This appearance seems in conflict with the general believed bowing effect, i.e. absorption edges should red shift when nitrogen composition increases. To interpret it, the Burstein-Moss effect should be taken into account because of the high carrier concentration in these InAsN samples, as can be seen in Table 2. In addition to the Burstein-Moss effect, the band gap renormalization effect should also be considered in this high carrier concentration case. The details of our calculation have been presented in section III. Table 2 shows our calculation result, the bottom of the conduction subband $E-$, the Fermi energy $E_{F}$, and the band gap renormalization $E_{B G R}$. We can see from this table that the calculated E- subband obeys bowing effect and we can note that the higher carrier concentrations cause higher $\mathrm{E}_{\mathrm{F}}$ and $\mathrm{E}_{\mathrm{BGR}}$.

Now we focus our attention on the PL spectrums of these InAs(N) samples as shown in Fig. 3. It can be seen from this figure that PL intensity degrades as the nitrogen composition increase. Two other points in this

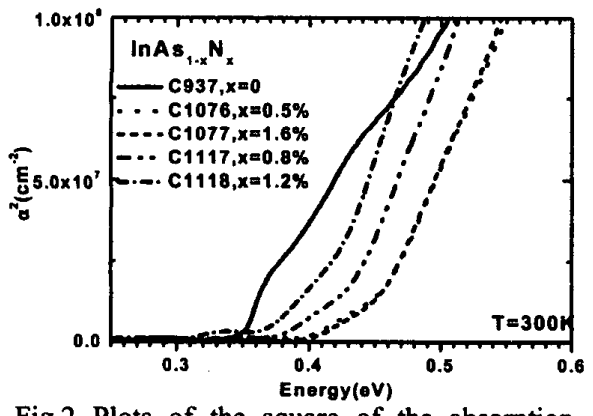

Fig.2 Plots of the square of the absorption coefficient $\left(\alpha^{2}\right)$ versus the photon energy deduced from $300 \mathrm{~K}$ IR transmission spectra.

Table 2 Carrier concentration, bottom of conduction

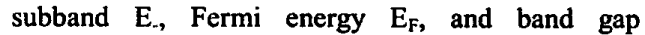
renormalization $E_{B G R}$. $E$. is relative to the top of valence band.

\begin{tabular}{cccccc}
\hline $\begin{array}{c}\text { Sample } \\
\text { No. }\end{array}$ & $\begin{array}{c}\text { Carrier } \\
\text { conc. } \\
\left(\mathrm{cm}^{-3}\right)\end{array}$ & $\begin{array}{c}\mathrm{N} \\
\text { comp. } \\
(\%)\end{array}$ & $\begin{array}{c}\mathrm{CB} \\
\text { subband } \\
\mathrm{E}- \\
(\mathrm{eV})\end{array}$ & $\begin{array}{c}\text { Fermi } \\
\text { energy } \\
\mathrm{E}_{\mathrm{F}} \\
(\mathrm{eV})\end{array}$ & $\begin{array}{c}\text { Band } \\
\text { renormal. } \\
\mathrm{E}_{\mathrm{BGR}} \\
(\mathrm{eV})\end{array}$ \\
\hline $\mathrm{C} 937$ & $2.64 \times 10^{16}$ & 0 & 0.352 & 0.005 & -0.007 \\
$\mathrm{C} 1076$ & $1.91 \times 10^{18}$ & 0.5 & 0.334 & 0.129 & -0.034 \\
$\mathrm{C} 1117$ & $1.21 \times 10^{18}$ & 0.8 & 0.331 & 0.106 & -0.028 \\
$\mathrm{C} 1118$ & $8.85 \times 10^{17}$ & 1.2 & 0.324 & 0.094 & -0.021 \\
$\mathrm{C} 1077$ & $3.24 \times 10^{18}$ & 1.6 & 0.302 & 0.157 & -0.038 \\
\hline
\end{tabular}

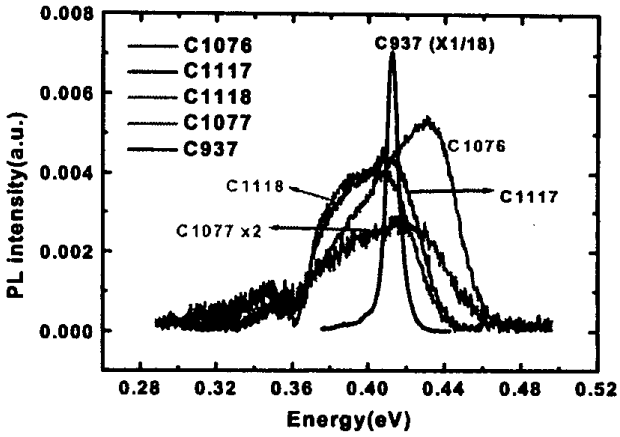

Fig.3 Low temperature (30K) PL spectrums of InAs and InAsN samples.

figure are worthy of note. First, the peak positions of these PL spectra show no bowing effect, neither. Second, PL spectrums of the InAsN samples except C937 all have very broad linewidths. We are interested in these phenomena because they seem to have some relations with the absorption edges in Fig. 2 and the Fermi energies in Table 2. Fig. 4 is a plot of PL linewidth and Fermi energy versus nitrogen composition. Here the definition of PL linewidth is different from the general one, i.e. full-width at half-maximum (FWHM), and is defined as full-width at one-tenth-maximum. From the figure we can see that PL linewidth is not dependent upon nitrogen composition but the Fermi energy. Trends of PL linewidth and Fermi energy are the same.

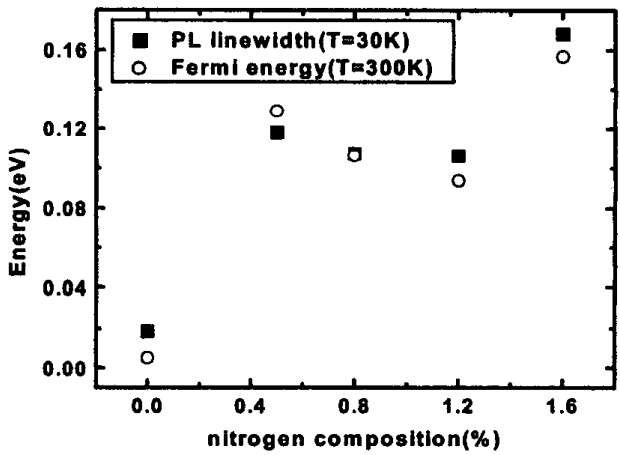

Fig.4 PL linewidth compared with Fermi energy.

To do a further investigation, the PL high-energy edges, defined as the energy where the intensity is one-tenth of the peak, are plotted in Fig. 5. For comparison, the absorption edges are also plotted in the same figure. It is clear in the figure that changes of these two energies are almost the same. The difference between them should be caused by temperature 


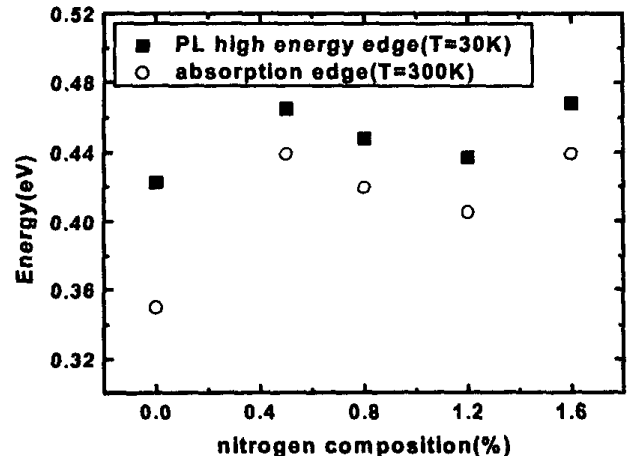

Fig. 5 PL high-energy edge compared with the absorption edge.

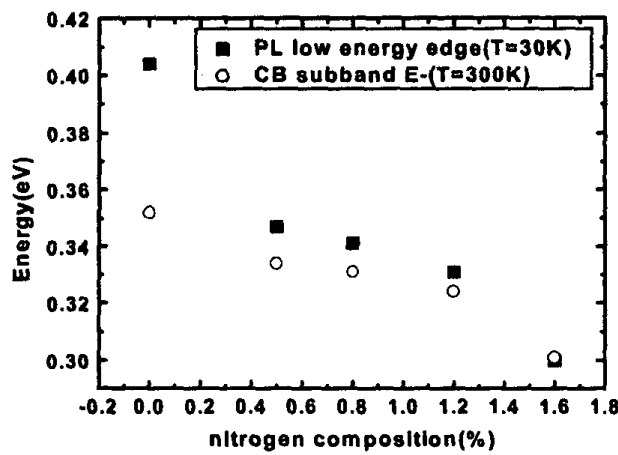

Fig. 6 PL low-energy edge compared with Esubband.

difference. On the other hand, the PL low-energy edges are plotted in Fig. 6 to compare with the calculated Esubband in Table 2. As can be seen, although the two results do not match well, they both follow the bowing effect. The energy difference between the E- subband and the PL low-energy edge is mainly due to the temperature difference and the band renormalization effect.

\section{Conclusions}

In this report, we use a method based on the band anticrossing model to calculate the band gap reduction in InAsN alloys. After considering the Burstein-Moss effect and the band renormalization effect, the bowing effect of InAsN alloy was evaluated. The material constant of the coupling element in the band anticrossing model, $\mathrm{C}_{\mathrm{NM}}$, obtained from the calculation is 1.68 . Moreover, it is found from the PL spectrums that the PL high-energy edges match with the absorption edges, and the low-energy edges are close to the calculated E- band gaps, and the broad linewidth of these spectrums are due to the high residual carrier concentration in the alloy.

\section{Acknowledgements}

This work was supported by the National Science Council and the Ministry of Education of the Republic of China under Contact No. NSC 90-2215-E-002-035- and 89-N-FA01-2-4-3, respectively.

\section{References}

1. S. Sakai, Y. Ueta, and Y. Terauchi, Jpn. J. Appl. Phys. 32, 4413 (1993).

2. S. H. Wei and A. Zunger, Phys. Rev. Lett. 76, 664 (1996).

3. L. Bellaiche, S. H. Wei, and A. Zunger, Phys. Rev. B 54, 17568 (1996).

4. W. Shan, W. Walukiewicz, J. W. Ager III, E. E. Haller, J. F. Geisz, D. J. Friedman, J. M. Olson, and S. R. Kurtz, Phys. Rev. Lett. 82, 1221 (1999).

5. Z. Pan, T. Miyamoto, D. Schlenker, S. Sato, F. Koyama, and K. Iga, J. Appl. Phys. 84, 6409 (1998).

6. M. R. Gokhale, J. wei, H. Wang, and S. R. Forrest, Appl. Phys. Lett. 74, 1287 (1999).

7. M. Kondow, K. Uomi, T. Kitatani, S. Watahiki, and Y. Yazawa, J. Cryst. Growth, 164, 175 (1996).

8. W. G. Bi and C. W. Tu, Appl. Phys. Lett. 70, 1608 (1997).

9. K. Iwata, H. Asahi, K. Asami, and S. Gonda, J. Cryst. Growth, 175/176, 150 (1997).

10. W. G. Bi and C. W. Tu, J. Appl. Phys. 80, 1934 (1996).

11. H. Naoi, Y. Naoi, and S. Sakai, Solid-state Electronics, 41, 319 (1997).

12. J. S. Wang, H. H. Lin, L. W. Sung and G. R. Chen, J. Vac. Sci. Technol. B, 19(1), 202, (2001).

13. G. R. Chen, H. H. Lin, J. S. Wang, and D. K. Shih, J. Appl. Phys. 90, 6230 (2001).

14. D. K. Shih, H. H. Lin, and Y. H. Lin, Electronics Lett., 37, 1342, (2001).

15. W. Shan, W. Walukiewicz, K. M. Yu, J. W. Ager III, E. E. Haller, Phys. Stat. Sol. (b) 223, 75 (2001).

16. C. Skierbiszewski, P. Wisniewski, W. Knap, and T. Suski, Appl. Phys. Lett. 76, 2409 (2000).

17. J. I. Pankove, Optical processes in semi-conductors, Prentice-Hall, Englewood Cliffs, NJ.1971.

18. D. C. Reynolds, D. C. Look, and B. Jogai, J. Appl. Phys. 88, 5760 (2000).

19. J. W. Matthews, and A. E. Blakeslee, J. Cryst. Growth, 27, 118 (1974).

20. M. A. Marciniak, R .L. Hengehold, Y. K Yeo, and G. W. Turner, J. Appl. Phys. 840, 480 (1998).

21. P. Dobrilla, and J. S. Blakemore, J. Appl. Phys. 58, 208 (1985) 